\title{
Controlled trial of natamycin in the treatment of allergic bronchopulmonary aspergillosis
}

\author{
D C Currie, C Lueck, H J Milburn, C Harvey, J L Longbottom, J H Darbyshire, \\ A J Nunn, P J Cole
}

\begin{abstract}
Allergic bronchopulmonary aspergillosis often requires treatment with oral corticosteroids to control the host response to Aspergillus fumigatus. In a double blind study 25 patients with allergic bronchopulmonary aspergillosis taking maintenance oral corticosteroids were randomly allocated to receive $5 \mathrm{mg}$ natamycin or placebo by nebuliser twice daily for one year. The primary aim of the study was to assess the steroid sparing potential of natamycin. Standardised reductions in corticosteroid dosage were therefore undertaken every five weeks, unless clinically contraindicated. Five patients were withdrawn in the first four months: two (1 natamycin, 1 placebo) died, two (1 natamycin, 1 placebo) had suspected drug reactions, and one (natamycin) was non-compliant. The pretreatment characteristics of the 20 patients (10 in each group) who completed the study were similar, 17 (9 natamycin, 8 placebo) having evidence of recent disease activity. At the end of the study prednisolone dose had been reduced by a similar amount in each group (median natamycin $2.25 \mathrm{mg}$, placebo $2.5 \mathrm{mg}$ ). Evidence of disease activity during the study year (transient shadowing on the chest radiograph, blood eosinophilia, or increases in antibodies to $A$ fumigatus, or any combination of these) was observed in similar numbers of patients in each group (5 natamycin, 7 placebo). There was no evidence that natamycin conferred benefit on these patients with allergic bronchopulmonary aspergillosis.
\end{abstract}

Prolonged oral corticosteroid treatment is often used in patients with allergic bronchopulmonary aspergillosis in an attempt to control the host inflammatory response to Aspergillus fumigatus, but corticosteroids are associated with significant morbidity. ${ }^{12} A$ fumigatus is found more frequently in the respiratory tract of patients with untreated allergic bronchopulmonary aspergillosis $(58 \%)$ and in larger quantities than in patients with other respiratory disease. ${ }^{34}$ Antifungal agents might therefore reduce the requirement for corticosteroids.

Nebulised antifungal agents, nystatin and natamycin (pimaricin), and nebulised brilliant green have been administered in an attempt to reduce the fungal load. ${ }^{5-7}$ The studies that have been reported were inadequately controlled and have shown equivocal evidence of benefit. In a group of eight patients with bronchopulmonary aspergillosis (initial culture positive for $A$ fumigatus) treated with nebulised natamycin $(2.5 \mathrm{mg}$ thrice daily for one month and thereafter twice daily) $A$ fumigatus disappeared from the sputum after a median of six (range 4-30) weeks. ${ }^{7}$ In a further study of four patients with allergic bronchopulmonary aspergillosis not receiving oral corticosteroids oral ketoconazole was associated with improvement in symptoms of asthma and a reduction in antibodies to $A$ fumigatus, ${ }^{8}$ but the risk of hepatotoxicity ${ }^{9}$ renders ketoconazole unsuitable as a long term alternative to corticosteroids.

In a pilot study six of seven patients with allergic bronchopulmonary aspergillosis treated with nebulised natamycin $(2.5 \mathrm{mg}$ thrice daily) and salbutamol were successfully withdrawn from maintenance corticosteroid treatment (5-20 mg prednisolone a day). We therefore set out to determine the corticosteroid sparing effect of natamycin in a controlled trial.

\section{Methods}

\section{STUDY DESIGN}

Patients were randomly allocated to inhale $5 \mathrm{mg}$ natamycin (Pimafucin, Brocades Ltd) or placebo $(0.9 \%$ saline $)$ delivered by nebuliser twice daily for 50 weeks in a double blind manner. The $0.5 \mathrm{ml}$ of trial solution $(5 \mathrm{mg}$ natamycin or placebo) was diluted with $3 \mathrm{ml}$ $0.9 \%$ saline and inhaled through a mouthpiece, an Inspiron nebuliser powered by a Portaneb 50 compressor being used. The random treatment allocation was stratified by severity of airflow obstruction and dose of oral corticosteroid at entry to the study according to the minimisation method. ${ }^{11}$

The primary aim of the study was to assess the corticosteroid sparing effect of natamycin. The corticosteroid dosage was therefore reduced every five weeks by one step according to a standard schedule unless this was clinically inappropriate. The steps in this schedule were $20,15,10,7 \cdot 5,6,5,4,3,2,1$, $0 \mathrm{mg}$ of prednisolone. Patients recorded their symptoms, use of the study drug and of beta agonist, and morning peak expiratory flow (best of three prebronchodilator results) on a diary card daily. The patients were seen, diary cards reviewed, and investigations performed 
Table 1 Pretreatment characteristics of the 20 patients with allergic bronchopulmonary aspergillosis completing the study

\begin{tabular}{|c|c|c|}
\hline & $\begin{array}{l}\text { Natamycin } \\
(n=10)\end{array}$ & $\begin{array}{l}\text { Placebo } \\
(n=10)\end{array}$ \\
\hline $\begin{array}{l}\text { Age (median (range), y) } \\
\text { Duration of the disease (median (range), y) }\end{array}$ & $\begin{array}{l}54 \cdot 5(37-74) \\
24 \cdot 5(9-38)\end{array}$ & $\begin{array}{l}48 \cdot 5(29-73) \\
17 \cdot 5(1-26)\end{array}$ \\
\hline No of acute exacerbations of chest symptoms in & & \\
\hline $\begin{array}{l}\text { preceding year (median (range)) } \\
\text { No of patients with disease activity }{ }^{\star} \text { in preceding five years }\end{array}$ & $3(1-12)$ & $\begin{array}{l}2(1-10) \\
8\end{array}$ \\
\hline $\begin{array}{l}\text { No or patients with aisease activity in preceang nve years } \\
\text { Transient radiographic shadows }\end{array}$ & 7 & 4 \\
\hline Blood eosinophilia $\left(>0.5 \times 10^{9} / 1\right)$ & 9 & 4 \\
\hline \multicolumn{3}{|l|}{ No of patients culture positive for Aspergillus fumigatus } \\
\hline $\begin{array}{l}\text { At enrolment } \\
\text { In the past }\end{array}$ & 1 & 1 \\
\hline In the past & 3 & 3 \\
\hline No of patients inhaling corticosteroids & 9 & 9 \\
\hline$\leqslant 1 \mathrm{mg}$ beclomethasone & 4 & 5 \\
\hline$>1 \mathrm{mg}$ beclomethasone & 5 & 4 \\
\hline
\end{tabular}

No statistically significant differences between the two groups.

$\star$ Defined as transient radiographic shadows or blood eosinophilia.

every five weeks. If the patient was stable or improving corticosteroid dosage was reduced. In the event of an acute exacerbation of respiratory symptoms, appropriate treatment was prescribed, usually antibiotics and bronchodilators with or without a short course of higher dose prednisolone. A short course of prednisolone was $30 \mathrm{mg}$ daily until a satisfactory clinical response had occurred, followed by slow reduction to a new maintenance dose one step higher on the standard schedule than before the exacerbation. The character of each exacerbation, the treatment given for it, and the total number of exacerbations for each patient were recorded.

PATIENTS

We studied 25 patients with allergic bronchopulmonary aspergillosis receiving maintenance oral corticosteroids. Patients with contraindications to the use of natamycin via nebuliser and those taking a fixed minimum dose of steroids were not included. Thirteen received natamycin and 12 placebo. All patients had a positive immediate skinprick test response to $A$ fumigatus, 24 had asthma (the remaining patient, in the natamycin group, had had bronchial plugs containing a mycelium of $A$ fumigatus and eosinophils); 24 had two or more episodes of shadowing visible on previous chest radiographs (the remaining patient, in the placebo group, had a single shadow associated with a heavy infiltrate of eosinophils in the bronchial wall); 22, 11 in each group, had fixed radiological abnormalities compatible with allergic bronchopulmonary aspergillosis and without a more likely cause, and 17 (12 natamycin, 5 placebo) had a record of previous blood eosinophilia ( $\left.>0.5 \times 10^{9} / 1\right)$ on one or more occasions. All 25 patients had IgE specific for $A$ fumigatus according to the radioallergosorbent test (RAST), and precipitins had been detected at some time in 24 patients (13 natamycin, 11 placebo).

In the 20 patients (10 natamycin, 10 placebo) who completed the study the pretreatment characteristics (medians and ranges in tables 1 and 2) in the two groups were similar; the natamycin group, however, were on average slightly older, had had symptoms for slightly longer, and had a slightly lower $\mathrm{FEV}_{1}$. Regular oral and inhaled drugs were not altered in the two months before the study or during the study except for oral corticosteroids as described above. Patients' compliance with the study treatment was assessed by monitoring drug use as recorded on diary cards and the degree of compliance described by each patient. In addition, the use of diluent for the study treatment was monitored by prescribing a known volume and requesting return of all diluent bottles. On the assumption that diluent not returned had been used appropriately, an objective percentage level of compliance was calculated, defined as $(100 \times$ observed volume of diluent used)/(expected volume).

The study was approved by the Brompton Hospital ethics committee.

\section{INVESTIGATIONS}

Sputum was examined every five weeks if the patient was expectorating. The character (purulent, mucopurulent, or mucoid) was recorded. A sample of the sputum was stained for eosinophils. The remainder of the specimen was diluted in an equal volume of Ringers' solution and homogenised by shaking with glass beads for 20 minutes. A sample of the homogenate was cultured for bacteria. The remainder was centrifuged at $2000 \mathrm{rev} /$ min for 10 minutes and the pellet plated on Sabouraud's medium and cultured for five days at $30^{\circ} \mathrm{C}$ for fungi. $\mathrm{FEV}_{1}$ and FVC were measured every five weeks, 20 minutes after $200 \mu \mathrm{g}$ inhaled salbutamol.

Plain posteroanterior chest radiography, a wet blood eosinophil count (eosinophilia defined as $\left.>0.5 \times 10^{9} / 1\right)$, RAST for IgE antibodies against $A$ fumigatus, ${ }^{12}$ and the enzyme linked immunosorbent assay (ELISA) for IgG antibodies to $A$ fumigatus ${ }^{12}$ were carried out every 10 weeks in all patients, and during acute exacerbations. Changes in

Table 2 Changes in corticosteroid dosage, lung function, and specific immunoglobulins during the study (medians with ranges in parenthesis)

\begin{tabular}{|c|c|c|c|c|}
\hline \multirow[b]{2}{*}{ Study week } & \multicolumn{2}{|c|}{ Natamycin $(n=10)$} & \multicolumn{2}{|c|}{ Placebo $(n=10)$} \\
\hline & 0 & 50 & 0 & 50 \\
\hline 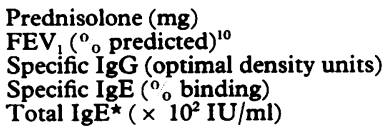 & $\begin{array}{l}6 \cdot 25(2-12 \cdot 5) \\
39(19-125) \\
0 \cdot 5(0 \cdot 1-1 \cdot 4) \\
25(19-33) \\
8 \cdot 1(2 \cdot 1-70)\end{array}$ & $\begin{array}{l}5 \cdot 5(0-10) \\
40(24-119) \\
0 \cdot 3(0 \cdot 1-1 \cdot 1) \\
20(12-30) \\
2 \cdot 4(1-45) \dagger\end{array}$ & $\begin{array}{l}7 \cdot 5(2-15) \\
58(28-102) \\
0 \cdot 7(0 \cdot 1-1 \cdot 1) \\
22(9-30) \\
12(1-45) \dagger\end{array}$ & $\begin{array}{l}4 \cdot 5(0-10) \\
62(28-92) \\
0 \cdot 6(0 \cdot 1-1 \cdot 5) \\
21(12-35) \\
5 \cdot 7(0 \cdot 45-65) \dagger\end{array}$ \\
\hline
\end{tabular}

Total IgG, IgA, and IgM were similar before and after treatment in the two groups and changed little.

No statistically significant differences between the two groups in pretreatment values or in changes during the study.

$\star$ Normal range $5-150 \mathrm{IU} / \mathrm{ml}$.

tone value missing. 
specific immunoglobulin concentrations to under $50 \%$ and over $150 \%$ of the initial value were arbitrarily defined as significant decreases and increases. Total immunoglobulins $\mathrm{G}, \mathrm{A}, \mathrm{M}$, and $\mathrm{E}$ were measured in all patients at the beginning and end of the study.

A full blood count, determination of urea and creatinine concentrations, liver function tests, urine analysis, and pure tone audiograms were performed before treatment, at 25 weeks, and at the end of the study to screen for adverse effects.

\section{ANALYSIS}

The data for the two groups were compared by means of the $\chi^{2}$ test and the Wilcoxon rank sum test for unpaired data.

\section{Results}

Five patients ( 3 natamycin, 2 placebo) were withdrawn from the study. Two patients, one in each group, with severe pre-existing obstructive airways disease died; one died two weeks after starting placebo and the other died after 11 weeks' natamycin, both with acute exacerbations of their chest disease. A third patient, receiving natamycin, developed wheeze and breathlessness that was related temporally to the study treatment and was withdrawn after 15 weeks. A fourth patient, receiving placebo, was withdrawn after 16 weeks during an acute exacerbation of the aspergillosis with haemoptysis attributed by the patient to the study treatment. The fifth patient, receiving natamycin, was withdrawn after five weeks for non-compliance with the nebuliser treatment. The results are presented for the 20 patients who completed the study.

Prednisolone dosage at 50 weeks was reduced by a similar amount in the two groups (median $2.25 \mathrm{mg}$ natamycin, $2.5 \mathrm{mg}$ placebo) and forced expiratory volume in one second $\left(\mathrm{FEV}_{1}\right)$ did not alter in either group (table 2). Transient chest radiographic shadows were observed on nine occasions (3 natamycin, 6 placebo) in seven patients (3 natamycin, 4 placebo). Blood eosinophilia was observed on 15 occasions (8 natamycin, 7 placebo) in seven patients (2 natamycin, 5 placebo). Specific IgE increased transiently in one placebo treated patient and decreased in one patient receiving natamycin. Specific IgG antibody increased transiently in one patient from each group. Overall, 12 patients (5 natamycin, 7 placebo) had transient radiographic shadows, blood eosinophilia, or an increase in specific immunoglobulins during the study. A fumigatus was cultured from sputum in only one patient in each group. There was a reduction in total IgE in both groups (table 2) but no other change in immunoglobulins.

The patterns of acute exacerbations were reviewed by scrutinising symptoms and signs recorded on the clinical forms and diary cards and the results of investigations. Individual exacerbations could not, however, be reliably categorised into the three expected typesasthma, allergic bronchopulmonary aspergil- losis, or acute bronchitis. The median number of exacerbations was similar in the two groups during the study year-natamycin 4.5 (range 1-10), placebo 5(1-13). The median number of days the patient was unwell was similar in the two groups-natamycin 22.5 (range 0-113), placebo 23 (5-56). The median number of courses of antibiotics was also similarnatamycin 2 (range $0-7$ ), placebo $2 \cdot 5(0-5)$-as was the number of high dose courses of corticosteroids-natamycin 1 (range 0-6), placebo 1 (0-3).

The nebuliser treatment was well tolerated, possible adverse effects occurring only in one patient from each group; both were withdrawn from the study. No significant changes were observed in the blood tests or urine analysis screening for adverse effects. No patient developed ototoxicity.

Eighteen of the 20 patients were more than $90 \%$ compliant with treatment as judged by the diary cards and volumes of returned diluent. The other two patients failed to return diluent bottles and their compliance as recorded on the diary cards was $82 \%$ and $85 \%$.

\section{Discussion}

Exacerbations of allergic bronchopulmonary aspergillosis occur sporadically throughout the year with an excess in the autumn and winter. ${ }^{4}$ $A$ fumigatus spore counts are greater in the autumn and winter ${ }^{13}$; possibly a critical fungal load is reached that triggers a host inflammatory response manifesting itself as an acute exacerbation.

The aim of our study was to reduce the need for corticosteroid suppression of the host inflammatory response by reducing the load of $A$ fumigatus in the lung with prolonged nebulised natamycin treatment. In patients with allergic bronchopulmonary aspergillosis the fungus may be absent from sputum, which makes measurement of the fungal load in the lungs impossible. Hyphae of $A$ fumigatus may not be present in the sputum during acute exacerbations in which $A$ fumigatus is subsequently shown to be present in the lungs. Substantial increases in total or specific IgE have been reported to be useful indicators of an acute exacerbation, ${ }^{14}{ }^{15}$ but in our study only one transient increase in specific IgE occurred despite radiographic evidence and blood eosinophilia suggesting allergic bronchopulmonary aspergillosis on several occasions.

The reductions of oral corticosteroids in the study. were carefully controlled. Inhaled corticosteroids, in the doses taken by the patients in this study, probably do not have an effect on exacerbations of the aspergillosis itself, ${ }^{16}$ but do improve control of asthma. Their dosage was not altered during the study. The patients in the study had a long history of allergic bronchopulmonary aspergillosis and required maintenance oral corticosteroids. Despite this treatment there were signs of recent disease activity in most patients. The low initial corticosteroid dosages, the low frequency of acute aspergillosis during the study year, and the small number of patients reduced the power of 
the study. The frequency of markers of inflammatory activity suggesting allergic bronchopulmonary aspergillosis and the frequency of all types of exacerbations were similar in the two groups during the study. The size of the reduction in oral corticosteroid dosage was similar in the two groups, while the degree of airflow obstruction remained constant. The only significant reductions in antibody levels were for total immunoglobulin $\mathrm{E}$ and these occurred in both groups. This finding contrasts with the reduction in IgG and IgE antibodies seen with ketoconazole treatment in the study of ketoconazole in allergic bronchopulmonary aspergillosis, ${ }^{8}$ though the total IgE and specific IgE levels before treatment in the four patients allocated to ketoconazole were considerably higher than those in the three placebo patients. ${ }^{8}$

Thus nebulised natamycin treatment for one year did not have a steroid sparing effect in patients with allergic bronchopulmonary aspergillosis whose disease was longstanding and relatively well controlled by oral corticosteroids, and from whose sputum $A$ fumigatus was seldom grown.

DC was supported by the Chest, Heart and Stroke Association. We thank Louise Taylor and Jill Price for their assistance and Brocades for supplying the active and placebo drug.

1 Maunsell K, Pearson RSB, Livingstone JL. Long-term corticosteroid treatment of asthma. Br Med J 1968; $661-5$.
2 Smyllie HC, Connolly CK. Incidence of serious complications of corticosteroid therapy in respiratory disease. Thorax 1968;23:571-81.

3 Pepys J, Riddell RW, Citron KM, Clayton YM, Short EI. Clinical and immunologic significance of Aspergillus fumigatus in the sputum. Am Rev Respir Dis 1959;80: 167-79.

4 McCarthy DS, Pepys J. Allergic bronchopulmonary aspergillosis. Clinical immunology: (1) Clinical features. Clin Allergy 1971;i:261-86.

5 McCarthy DS, Robertson DG. Antifungal agents in bronchopulmonary aspergillosis. Lancet 1968;i:1089-90.

6 Henderson AH, Pearson JEG. Treatment of bronchopulmonary aspergillosis with observations on the use of natamycin. Thorax 1968;23:519-23.

7 Edwards G, La Touche CIP. The treatment of bronchopulmonary mycoses with a new antibiotic-pimaricin. Lancet 1964; i:1349-53.

8 Shale DJ, Faux JA, Lane DJ. Trial of ketoconazole in noninvasive pulmonary aspergillosis. Thorax 1987;42:26-31.

9 Lake-Bakaar G, Scheuer PJ, Sherlock S. Hepatic reactions associated with ketoconazole in the United Kingdom. Br Med J 1987;294:419-22.

10 Cotes JE. Lung function: principles and application in medicine. 4th ed. Oxford: Blackwell, 1979:365-80.

11 Pocock SJ. Clinical trials: a practical approach. Chichester: Wiley, 1983:84-7.

12 Longbottom JL. Applications of immunological methods in mycology. In: Weir OM, Herzenberg LA, Blackwell C, Herzenberg LA, eds. Handbook of experimental immunology. Vol 4. 4th ed. Oxford: Blackwell, 1986:121.1-30.

13 Mullins J, Harvey R, Seaton A. Sources and incidence of airborne Aspergillus fumigatus (Fr-es). Clin Allergy 1976;6:209-17.

14 Rosenberg $M$, Patterson $R$, Roberts $M$. Immunologic responses to therapy in allergic bronchopulmonary aspergillosis: serum IgE value as an indicator and predictor of disease activity. J Paediatr 1977;91:914-7

15 Imbean SA, Nichols D, Flaherty D, Dickie H, Reed C. Relationships between prednisone therapy, disease activity, and the total serum IgE level in allergic bronchopulmonary aspergillosis. J Allergy Clin Immunol 1978;62:91-5.

16 Research Committee of the British Thoracic Association Inhaled beclomethasone dipropionate in allergic bronchopulmonary aspergillosis. Br J Dis Chest 1979;73. 349-56. 